\title{
La Psicología en Bolivia: formación y situación laboral.
}

\section{Psychology in Bolivia: training and employment.}

Fecha de recepción: 04/11/2014

Fecha de aceptación: 18/06/2015
Marion K. Schulmeyer

Universidad Privada de Santa Cruz de la Sierra.

\section{resumen/abstract:}

A partir de la realidad socioeconómica del país, se presenta algunos indicadores de la educación superior en Bolivia, tanto pública como privada, para después ahondar en las características de los programas de Psicología en la actualidad, a nivel de contenidos de pregrado y programas de postgrado, y se mencionan los sistemas de evaluación de la calidad universitaria en Bolivia como algo de reciente aplicación en las carreras de psicología. Asimismo, se muestra la situación legal y laboral de los psicólogos haciendo un breve recorrido por los caminos que se están abriendo desde las distintas áreas de desempeño profesional, y presentando las asociaciones que los agrupan y fomentan el desarrollo de la disciplina en el país. Finalmente se analizan los retos que enfrenta la profesión, la formación universitaria y por ende, la formación docente y la psicología como ciencia, en un país donde la investigación es un enunciado no una realidad. ${ }^{1}$

Beginning with a short review of Bolivia's socio-economic context, we present a few indicators of the public and private universities in the country and review the contents of psychology pre-graduate programs, the graduate programs offered, and the recent development of quality evaluation systems in Bolivian universities. We also consider the job market and legal status of Psychology, with a brief glance at new opportunities that are emerging for different professional areas and the associations that group and develop the discipline in the country. Finally we explore the challenges faced in this context as a profession, in university education, therefore in faculty training, and as a science, in a country where research is a statement not a reality.

\section{palabras clave/keywords:}

psicología, formación, empleo, investigación

psychology, training, employment, research,

\footnotetext{
${ }^{1}$ Se agradece a la Prof ${ }^{a}$. Juana Haydeé López Vargas, Psicóloga Social de la Universidad Juan Misael Saracho, de Tarija, y al coordinador del Instituto de Investigaciones en Ciencias del Comportamiento de la Universidad Católica Boliviana "San Pablo", de La Paz, Dr. Bismarck Pinto, por su apoyo e información.
}

Al hablar de los procesos de formación de la Psicología en los distintos países latinoamericanos, no es extraño encontrar una contextualización de la precariedad social y económica que determina el contexto en el que se da dicha formación. Por lo que situar Bolivia, en la realidad latinoamericana puede ayudar a comprender el desarrollo y contexto actual de la Psicología en este país. Además de los aspectos históricos de inestabilidad política que caracterizaron el país hasta 1984, y que afectaron al inicio de las primeras carreras de Psicología en los años 70, un factor que consideramos decisivo es el económico, ya que determina los niveles de educación, acceso a servicios de salud y bienestar general. En este sentido nos 
pareció adecuado observar el Índice de Desarrollo Humano de los países sudamericanos, ya que este indicador social se basa en la esperanza de una vida larga y saludable, los niveles de educación y la consideración de un nivel de vida digno. Como se puede observar en la Tabla 1, Bolivia junto a Paraguay ocupan las últimas posiciones del IDH sudamericano, con el agravante, en el caso de Bolivia, de tener un $60 \%$ de su población debajo de la línea de pobreza (PNUD, 2013; Rodríguez y Weise, 2006).

\begin{tabular}{|c|c|c|c|}
\hline País & Posición IDH & $\begin{array}{c}\text { Ingreso Nacional } \\
\text { Bruto per cápita } \\
\text { (\$us) }\end{array}$ & $\begin{array}{l}\text { \% de población bajo } \\
\text { la línea de pobreza }\end{array}$ \\
\hline \multicolumn{4}{|l|}{ IDH muy alto } \\
\hline Chile & 40 & 14987 & --- \\
\hline Argentina & 45 & 15347 & --- \\
\hline \multicolumn{4}{|l|}{ IDH alto } \\
\hline Uruguay & 51 & 13333 & 18.1 \\
\hline Venezuela & 71 & 11475 & --- \\
\hline Perú & 77 & 9306 & --- \\
\hline Brasil & 85 & 10152 & 21.4 \\
\hline Ecuador & 89 & 7477 & --- \\
\hline Colombia & 91 & 8711 & 37.2 \\
\hline \multicolumn{4}{|l|}{ IDH medio } \\
\hline Bolivia & 108 & 4444 & 60.1 \\
\hline Paraguay & 111 & 4497 & 34.7 \\
\hline
\end{tabular}

(PNUD, 2013)

En este panorama, la realidad salarial de los psicólogos como profesionales del ámbito de la salud, resulta bastante bajo. Por ejemplo, según datos del 2012, el salario mínimo nacional, era de \$us. 144, y la remuneración promedio para las distintas actividades nacionales era de \$us. 490. El salario promedio más alto era el que recibía el sector de producción de energía eléctrica, gas y agua (\$us 1222), mientras que el salario para el sector de Servicios Sociales y de Salud - donde se sitúan los psicólogos - era de \$us. 330. Debajo del sector de salud estaban los obreros de las maquilas, madera y el personal de restaurantes, bares y cantinas (Datax, 2012). Por tanto, salvo excepciones, la mayor parte de los psicólogos en Bolivia, cuentan con un salario que cubre sus necesidades básicas, y por tanto, tienen pocas posibilidades de continuar su formación una vez graduados, acceder a bibliografía actualizada, adquirir herramientas necesarias para desarrollar su trabajo - como pueden ser los tests - o 


\section{dossier: Psicología lberoamericana - |I}

Marion K. Schulmeyer

participar de eventos científicos dentro y fuera del país. Lo que agrega a la mediterraneidad geográfica del país, un factor más de enclaustramiento.

\section{Educación Superior y Carreras de Psicología}

En este contexto, aunque la educación superior en las universidades públicas es prácticamente gratuita, solo hay un 39\% de matrícula en formación universitaria (Fundapro, 2011, PNUD, 2013), y aunque no hay datos nuevos, los estudios existentes estimaban que a finales de los 90 la deserción rondaba el $77 \%$ y que solo un $2 \%$ se titulaba ${ }^{1}$ (Centro Interuniversitario de Desarrollo, 2006). En estudios algo más recientes se observó que los motivos de deserción son principalmente económicos (Rivera, 2006). Esto se explica cuando se estudia la composición sociocultural de los estudiantes de las universidades públicas, ya que del 60 al 90 por ciento - dependiendo de la universidad - procede de colegios públicos que brindan educación gratuita (Rodríguez y Weise, 2006), así como los motivos por los que muchos se matriculan en estas universidades, que según un estudio de la Fundación para la Producción no se trata tanto de razones académicas, sino del acceso a un seguro médico, al comedor universitario y a los precios preferenciales en el transporte (Fundapro, 2011). Estos beneficios, más la dificultad de hacer coincidir el trabajo con la universidad, también contribuyen a entender por qué los índices de graduación son bajos y toma un promedio de ocho a 13 años el graduarse (Centro Interuniversitario de Desarrollo, 2006; Rodríguez y Weise, 2006).

Actualmente, hay una población de alrededor de 470000 estudiantes universitarios, en las 59 universidades públicas y privadas que están en funcionamiento en el país (INE, 2015). Las universidades públicas son autónomas, funcionan con financiamiento estatal y son coordinadas por el Comité Ejecutivo de la Universidad Boliviana (CEUB) y las privadas funcionan con ingresos propios y son supervisadas por el Ministerio de Educación. Cinco de las universidades públicas y 12 privadas imparten la carrera de Psicología, habiendo 27 carreras de Psicología en funcionamiento porque algunas universidades privadas tienen sedes en varios departamentos de Bolivia.

No se encontraron datos actualizados, de acceso público que permitan saber cuántos son todos los estudiantes de psicología inscritos o graduados de las universidades públicas y privadas de Bolivia. Pero por datos del Ministerio de Educación (2011), se sabe que del 2001 al 2009, se inscribieron 4056 estudiantes a la Carrera de Psicología en las Universidades Privadas bolivianas (70\% mujeres, 30\% varones) y durante esos años, se graduaron 709 psicólogos ( $82 \%$ mujeres $18 \%$ varones). Tomando en cuenta estos datos, si las universidades públicas y privadas graduasen un porcentaje similar de estudiantes, cabría esperar que entre 2001 y 2009, en Bolivia se hubiera titulado alrededor de 2200 psicólogos nuevos. Pero, no hay consenso en las cifras de graduación. Por ejemplo, según la Fundación para la Producción (2011) la relación entre titulados y matriculados es de dos a diez en las universidades públicas y de cuatro a diez en las privadas, pero según Rodríguez y Weise, (2006) la universidad pública titula un mayor porcentaje de estudiantes que la universidad privada, mientras que Calderón, en 1999, hablaba de 19 a 47 por ciento de titulados en los programas de Psicología cuando en esas fechas el porcentaje de titulados de las universidades públicas

\footnotetext{
${ }^{1}$ Se cree que estas cifras mejoraron en los últimos años con el posicionamiento de las universidades privadas y los nuevos mecanismos de graduación de las universidades públicas que dieron opción a graduación sin tesis a través del acceso a cursos de postgrado y otros.
} 


\section{dossier: Psicología lberoamericana - ||}

La Psicología en Bolivia: formación y situación laboral

rondaba el $2 \%$, probablemente sus cifras se basaron en los indicadores de la Universidad Católica, lo que de ser así, coincide con los resultados que Fundapro obtiene en el 2011.

Lo que sí ha venido pasando, es que desde que comenzaron a existir las universidades privadas en los años 80, progresivamente fue aumentando el número de estudiantes en ellas, recibiendo aproximadamente un 43 por ciento de toda la matrícula de estudiantes nuevos del país (Fundapro, 2011; INE, 2012). A esta tendencia se suman las carreras de Psicología del ámbito privado recién a partir de los años 90 . Porque hasta finales de los años 80 solo había cuatro carreras de psicología en funcionamiento. La primera, de la Universidad Católica Boliviana "San Pablo" (universidad privada que goza de una situación particular ya que responde al CEUB) y tres más, en universidades públicas. En 1992 comienzan a funcionar las dos primeras carreras de Psicología en universidades privadas y a partir de entonces poco a poco, se van aprobando nuevas carreras hasta el 2008.

A nivel de licenciatura, las carreras han tenido de ocho a diez semestres de duración y, en general, han brindado una formación generalista y profesionalizante. Tal vez, en un par de universidades se pueda encontrar una perspectiva científico-profesional, pero actualmente resulta difícil reconocerlo en base a los Planes de Estudio existentes. Poco se puede decir de la orientación que imprimieron en sus primeros diseño curriculares ya que no se tiene registro de ello, salvo por los trabajos de Aguilar (1983), Calderón (1999) y Vía (2000) que permiten conocer a profundidad el desarrollo de la carrera de Psicología de la Universidad Católica Boliviana, y el inicio de las primeras carreras en las universidades públicas. En estas últimas, al parecer, la orientación dependió de la formación de los docentes y directores del momento, salvo posibles excepciones, como la carrera de la Universidad Mayor de San Simón, en Cochabamba, en la que el psicoanálisis tuvo desde el inicio un papel determinante.

Para saber cómo se está impartiendo la Psicología en la actualidad, se analizó los Planes de Estudio en ejecución de 13 Universidades Privadas y cuatro Universidades Públicas. Se organizó las materias en 16 categorías en base a las categorías expuestas por Puche (2003) y a las características propias de los programas bolivianos (Schulmeyer, 2014a). No se encontró diferencias estadísticamente significativas entre las frecuencias de materias de las distintas áreas de la Psicología en los programas de las universidades públicas y privadas (en todos los casos $\chi^{2}$ tuvo una $p>0,05$ ), pero de todas maneras, después de describir el énfasis que se da a las diferentes áreas de estudio, mencionaremos en cuáles tienden a diferenciarse (ver Tabla 2).

Generalmente, los planes de estudio dedican un 9\% de sus materias al área metodológica, $10 \%$ al área de evaluación, 25\% a las distintas teorías psicológicas (psicoanálisis, cognitivoconductual, sistémica, humanista) y $29 \%$ al área de aplicación (educativa, clínica, jurídica y organizacional).

En relación a la presencia de las distintas teorías psicológicas, se encuentra una mayor concentración de materias de orientación cognitivo-conductual en las universidades privadas y psicoanalíticas en las universidades públicas. Sin embargo, es posible que si se analizara los programas de las materias (Psicología General, Psicología del Desarrollo, Psicopatología,

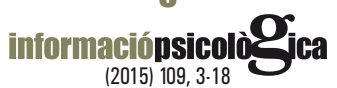


Tabla 2. Áreas de formación en las universidades bolivianas

\begin{tabular}{|c|c|c|c|c|c|c|}
\hline \multirow[t]{2}{*}{ Área de Formación } & \multicolumn{2}{|c|}{$\begin{array}{c}\text { Universidades } \\
\text { Privadas }\end{array}$} & \multicolumn{2}{|c|}{$\begin{array}{c}\text { Universidades } \\
\text { Públicas }\end{array}$} & \multicolumn{2}{|c|}{ Total } \\
\hline & $n$ & $\%$ & $n$ & $\%$ & $n$ & $\%$ \\
\hline Evaluación & & & & & & 10 \\
\hline Proyectivas & 17 & 3 & 7 & 3 & 24 & 3 \\
\hline Psicométricas & 15 & 3 & 4 & 2 & 19 & 2 \\
\hline Psicodiagnóstico & 11 & 2 & 5 & 2 & 16 & 2 \\
\hline Evaluación Psicológica & 6 & 1 & 6 & 3 & 12 & 2 \\
\hline Teoría Psicométrica & 3 & 1 & 0 & 0 & 3 & 0 \\
\hline Entrevista & 3 & 1 & 1 & 0 & 4 & 1 \\
\hline Metodología, Diseños & 31 & 5 & 15 & 7 & 46 & 6 \\
\hline Estadística & 17 & 3 & 7 & 3 & 24 & 3 \\
\hline Historia y Sistemas Psicológicos & 18 & 3 & 8 & 4 & 26 & 3 \\
\hline Bases biológicas & 31 & 5 & 15 & 7 & 46 & 6 \\
\hline Psicología evolutiva & 30 & 5 & 7 & 3 & 37 & 5 \\
\hline Psicología social, comunitaria & 36 & 6 & 19 & 9 & 55 & 7 \\
\hline Teorías psicologicas & & & & & & 25 \\
\hline Psicoanálisis & 14 & 2 & 9 & 4 & 23 & 3 \\
\hline Humanista & 5 & 1 & 5 & 2 & 10 & 1 \\
\hline Cognitivo-conductual & 37 & 6 & 10 & 5 & 47 & 6 \\
\hline Sistémica & 9 & 2 & 4 & 2 & 13 & 2 \\
\hline Procesos psicologicos básicos & 15 & 3 & 6 & 3 & 21 & 3 \\
\hline Fundamentos interdisciplinarios & 31 & 5 & 17 & 8 & 48 & 6 \\
\hline Personalidad & 7 & 1 & 4 & 2 & 11 & 1 \\
\hline Psicopatología & 22 & 4 & 9 & 4 & 31 & 4 \\
\hline Áreas de aplicación & & & & & & 20 \\
\hline Clínica y de la salud & 37 & 6 & 10 & 5 & 47 & 6 \\
\hline Organizacional & 45 & 8 & 5 & 2 & 50 & 6 \\
\hline Educativa & 52 & 9 & 16 & 7 & 68 & 9 \\
\hline Jurídica & 4 & 1 & 1 & 0 & 5 & 1 \\
\hline Ética & 9 & 2 & 2 & 1 & 11 & 1 \\
\hline Otras áreas de aplicación & 51 & 9 & 19 & 9 & 70 & 9 \\
\hline Materias de apoyo & 39 & 7 & 11 & 5 & 50 & 6 \\
\hline
\end{tabular}

(Schulmeyer, 2014a)

etc.) se encontraría que un gran porcentaje de ellas se dicta desde una perspectiva psicoanalítica porque es difícil encontrar profesionales con experiencia formados desde otras perspectivas teóricas. Asimismo, en este momento existen muy pocas materias de orientación humanista o sistémica en los programas.

Acerca de las áreas de aplicación, se encuentra que el área educativa seguida del área clínica, tiene mucho peso en los programas, (incluso al analizar los contenidos de las materias del área de evaluación, el énfasis se da en este sentido), (Schulmeyer, 2014b). La alta fre- 
cuencia de materias en el área organizacional se debe a una universidad privada que brinda esta orientación formativa por lo que no se la considera como una diferencia representativa de la formación en general. No obstante, llama la atención la poca presencia de materias del área jurídica/forense, sobre todo en las universidades públicas, ya que es un área en el que se requiere cada vez más personal especializado, y que además, absorbe sus graduados. Así también, la poca presencia de materias exclusivas del área de la Salud, siendo la Psicología una carrera reconocida por el Ministerio de Salud como perteneciente al área.

Sí hay una presencia un poco mayor de materias de Psicología Social, Psicología Comunitaria y de Fundamentos Interdisciplinarios como Antropología y Sociología, en las universidades públicas, además de la enseñanza de lengua aimara y quechua como parte del Plan de Estudios en las ciudades donde hay una mayor concentración poblacional de estas etnias. Lo que es de esperar por la orientación política de la universidad pública y las áreas de aplicación (clínica, educativa y social) que las Carreras de Psicología definieron para sus Planes de Estudio (CEUB, 1997; Weise, 2005). Pero, sorprende que el énfasis en el área social no sea más evidente porque como ya se dijo, la diferencia entre universidades públicas y privadas no es significativa. Uno de los factores que puede explicar esta situación es la fuerza de los psicólogos clínicos en las universidades que ha dificultado el desarrollo de los psicólogos sociales (J. López, comunicación personal, 14 de enero de 2013).

Ahora bien, la formación del plantel docente también es interesante de analizar, aún con las limitaciones de información existentes. Según datos de 1998, el 82\% de los docentes universitarios eran licenciados, $5 \%$ tenía una especialidad, $8 \%$ maestría y el $1 \%$ doctorado. Estos porcentajes con seguridad mejoraron en los últimos años porque la Universidad Publica definió que los docentes debían tener una especialidad en Educación Superior y el Reglamento de Universidades Privadas exige lo mismo y prácticamente en todas las universidades se ofrecen maestrías en Educación Superior (Rodríguez y Weise, 2006). Además se impartieron algunos programas de Maestría en distintas áreas de la Psicología y un programa de doctorado que con seguridad cambió estos indicadores.

Por ejemplo, el caso de los docentes de Psicología en algunas universidades dista mucho de los indicadores nacionales. Así, en la actualidad, la Carrera de Psicología de la Universidad Privada de Santa Cruz de la Sierra cuenta con un plantel docente con 36\% de doctorados, $36 \%$ con maestría y $28 \%$ con licenciatura y diplomado en Educación Superior (estos indicadores son superiores a los de otras carreras en la misma universidad).

\section{Postgrado}

El primer programa de postgrado en Bolivia lo realizó la Universidad Mayor de San Simón, en 1974, con una Especialidad en Ginecología y el segundo, la Universidad Mayor de San Andrés, en 1984, en Ciencias del Desarrollo. Pero hasta los años 90 la formación postgradual en el país fue reducida (Rodríguez y Weise, 2006).

Entre los programas de postgrado en el sector público y en el privado hay unos que otorgan grado y otros que no. Los programas de "postítulo" y los "diplomados" no otorgan grado. Los primeros no contemplan un número de horas definido y los segundos deben tener al me-

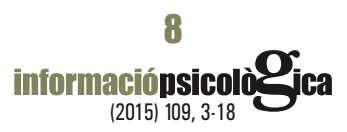


nos 200 horas académicas. Después están los grados de Especialidad, Maestría y Doctorado (Rodríguez y Weise, 2006).

Según el informe de los programas ofrecidos por las universidades bolivianas, publicado el 2011 por el Ministerio de Educación, se ofrecieron en algún momento siete programas de Maestría en el área de la Psicología. La información no especifica si realmente se llegaron a realizar o no. De hecho, más de uno de los mencionados se abrió pero no concluyó, y otros se dictaron una vez y se descontinuaron (ver Tabla 3 ).

Tabla 3. Programas de postgrado en universidades bolivianas

\begin{tabular}{|c|c|c|c|}
\hline Universidad & Programa & $\begin{array}{c}\text { Grado } \\
\text { Académico }\end{array}$ & $\begin{array}{l}\text { Año de } \\
\text { creación }\end{array}$ \\
\hline \multirow{3}{*}{$\begin{array}{l}\text { Universidad } \\
\text { Autónoma Gabriel } \\
\text { René Moreno }\end{array}$} & Psicología Organizacional & Maestría & \\
\hline & Neuropsicología & Maestría & \\
\hline & Psicología Jurídica Forense & Maestría & \\
\hline Universidad & Psicología Comunitaria y de la Salud & Maestría & \\
\hline $\begin{array}{l}\text { Autónoma Juan } \\
\text { Misael Saracho }\end{array}$ & Psicología Jurídica y Forense & Maestría & \\
\hline $\begin{array}{l}\text { Universidad Privada } \\
\text { del Valle }\end{array}$ & Psicología Social & Maestría & $\begin{array}{l}1999 \\
2005\end{array}$ \\
\hline \multirow{4}{*}{$\begin{array}{l}\text { Universidad } \\
\text { Católica Boliviana } \\
\text { "San Pablo" }\end{array}$} & Clínica Sistémica y Terapias Breves & Especialidad & \\
\hline & Psicología Laboral & Especialidad & \\
\hline & $\begin{array}{l}\text { Clínica Psicoanalítica e Intervención } \\
\text { en Crisis }\end{array}$ & Especialidad & \\
\hline & Psicología de la Salud & Maestría & \\
\hline $\begin{array}{l}\text { Universidad Privada } \\
\text { de Santa Cruz de la } \\
\text { Sierra }\end{array}$ & $\begin{array}{l}\text { Terapia Sistémica con Familias y } \\
\text { Parejas (con el Instituto de Terapia } \\
\text { Familiar de Londres) }\end{array}$ & Especialidad & 1999 \\
\hline
\end{tabular}

(Ministerio de Educación, 2011; UPSA, 2000)

Con seguridad se realizaron programas a nivel de Diplomado y Especialidad que no figuran en la Tabla 3 ya que las universidades públicas al ser autónomas no tienen por qué informar sobre los programas que realizan, a menos que deseen hacerlo, y antes del 2013, las privadas solo debían hacer aprobar sus planes de maestría e informar sobre los otros programas, los cuales al parecer el Ministerio decidió no incluir en la Guía del 2011. 
Hay que destacar la contribución de la Universidad Católica Boliviana "San Pablo" a la formación postgradual en Psicología. Actualmente se está ofreciendo la décima versión de la Especialidad en Clínica Sistémica y tercera versión de la Maestría en Psicología de la Salud. En el 2000, en convenio con la Universidad de Granada, impartió un Doctorado en Psicología Social, Aplicaciones y Métodos que contó con dos menciones: Psicología de la Salud y Psicología Organizacional. Cuando la carrera de psicología cumplió 38 años, en el 2009, ofreció el segundo Programa de Doctorado en Psicología que ayudó a elevar los niveles de formación de los docentes de Psicología, sobre todo, en La Paz (B. Pinto, comunicación personal, 9 julio 2014).

\section{Sistemas de Evaluación de la Calidad Universitaria}

El interés por desarrollar sistemas de evaluación en las universidades comenzó en los años 90. En junio de 1993 se realizó un Seminario Internacional de Educación Superior en el que se señalaron las insuficiencias y desafíos de la educación superior boliviana, a raíz del cual se vio la necesidad de crear, desde el Estado, un Consejo Nacional de Evaluación y Acreditación de la Calidad de la Educación Superior (CONAMED), como parte de la Ley de Reforma Educativa (Ley 1564). Este organismo debía hacerse cargo de la acreditación de universidades públicas y privadas, condicionando la asignación de fondos a las públicas en función a los resultados de la evaluación. Obviamente la iniciativa fue resistida y no se llevó a cabo (Herrera, 1994; Rodríguez y Weise, 2006). De ahí en adelante los procesos de evaluación y acreditación de universidades públicas y privadas se pusieron en marcha por separado.

Las universidades públicas crearon instancias internas de evaluación, y a nivel nacional, el CEUB coordina los procesos de evaluación externa y acreditación. En un primer momento se evaluaron las carreras de ingeniería y medicina. Pero en los últimos años, el Sistema de la Universidad Pública, con representantes de todo el país, definió criterios para evaluar las Carreras de Psicología a través de la Secretaría Nacional de Evaluación y Acreditación del CEUB, y en el 2011 comenzó a realizar evaluaciones externas de algunas carreras de Psicología (CEUB, 2014). Todavía es pronto para saber si estos procesos lograran incentivar una preocupación genuina por la calidad y el mejoramiento continuo, y menos aún para saber si esto va a tener un impacto positivo en los recursos de apoyo a la docencia y la investigación.

Por otra parte, los procesos de evaluación de las universidades privadas las realiza el Ministerio de Educación Superior, haciendo la primera evaluación en 1997, en la que evaluó las condiciones en las que funcionaban las universidades privadas, sin que existiera todavía un marco normativo para reglamentarlas ${ }^{2}$. A partir del 2001, ya con un Reglamento de Universidades Privadas aprobado, el Ministerio realizó una segunda evaluación en la que las universidades podían ser reconocidas por el Estado como Universidades Plenas (Rodríguez y Weise, 2006).

Por su parte, algunas universidades privadas interesadas en monitorear su calidad buscaron entidades internacionales de evaluación y acreditación. Como es el caso de la Universidad

${ }^{2}$ En base a los resultados de esta evaluación, se encuentra una reflexión interesante sobre el estado de las universidades privadas en Contreras (1998). 
Privada de Santa Cruz de la Sierra que se somete regularmente a procesos de acreditación con el Centro Interamericano de Desarrollo Académico (CINDA) y la Red Latinoamericana de Cooperación Universitaria (RLCU) para asegurar sus estándares de calidad (UPSA, 2014). Pero ninguna participó en procesos específicos para evaluar carreras, salvo por los procesos de evaluación de Mercosur, los cuales todavía no incluyen la Carrera de Psicología.

\section{Mercado Laboral}

En general, la formación no acompaña las necesidades del mercado laboral, y las carreras de Psicología no son la excepción. Asimismo, la formación a nivel postgradual sigue siendo limitada, lo que disminuye las posibilidades de acceder y/o exigir mejores salarios, ya que existe cierta congruencia entre niveles de formación y mejoras salariales (Fundapro, 2011).

En este momento, el campo de trabajo de los psicólogos en Bolivia va haciéndose cada vez mayor en el área educativa, organizacional y jurídica, mientras que el área de la salud es todavía la que más resistencia encuentra. Aunque cada vez hay más espacios para los psicólogos, el estudio de Fundapro (2011) muestra que en la mayor parte de las regiones la oferta de psicólogos es mucho mayor a la demanda que hay (ver Figura 1).

Figura 1. Estructura de la demanda vs. oferta de psicólogos en Bolivia

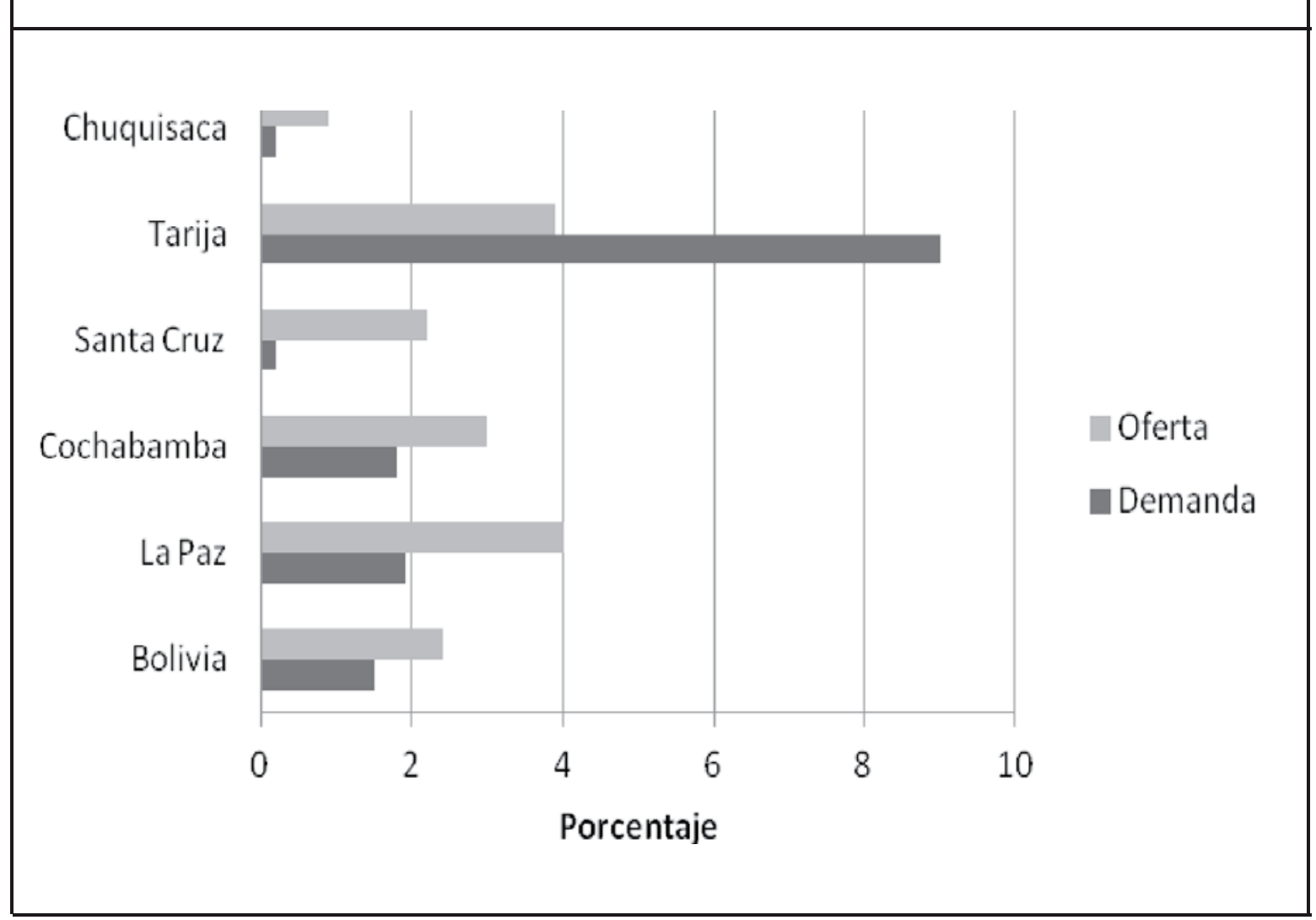




\section{dossier: Psicología lberoamericana - ||}

La Psicología en Bolivia: formación y situación laboral

Los cinco departamentos que figuran en el gráfico concentran el 93\% de las Carreras de Psicología. La descomunal diferencia que se observa en los datos de Tarija, puede deberse a que al ser un departamento productor de petróleo, en los últimos años, se pudo haber beneficiado por el alza del precio y demanda de materias primas, lo que impulsó un veloz crecimiento económico que pudo afectar positivamente el mercado laboral.

\section{Asociación y Regulación}

La Psicología en Bolivia está organizada, informalmente, en Colegios Profesionales y Asociaciones.

A mediados de los años 70, se creó el Colegio de Psicólogos Nacional en La Paz, y en 1987 se propuso una Ley del Ejercicio Profesional del Psicólogo, la cual nunca se logró hacer aprobar (Aguilar, 1983; Brañez, 2012, Calderon, 1999). En la actualidad, el Colegio Nacional existe nominalmente, pero en realidad, funcionan en mayor o menor medida, los colegios departamentales. Hay Colegios de Psicólogos en los seis departamentos del país que cuentan con carreras de Psicología (Santa Cruz, Cochabamba, La Paz, Tarija, Chuquisaca y Oruro) y uno menos conocido, en Potosí.

La afiliación a los Colegios de Psicólogos es voluntaria porque no hay una ley que regule la profesión, sin embargo, diversas instituciones departamentales y nacionales reconocen su existencia. Por ejemplo, los Colegios de Psicólogos participan con un evaluador externo en las defensas de tesis de las universidades privadas a solicitud del Ministerio de Educación, responden a solicitudes del poder judicial emitidas a través de jueces y fiscales, emiten certificados de afiliación para instituciones que exigen la colegiatura como requisito de contratación (al menos en el departamento de Santa Cruz), y en algunos casos, intervienen en procesos éticos que involucran a sus afiliados. Pero, no pueden emitir juicio alguno sobre profesionales no afiliados, a menos que sea solicitado expresamente por el poder judicial (lo cual por ahora no ha sucedido). En definitiva, los colegios profesionales existen por voluntad de la sociedad civil y las instituciones departamentales que consideran que son necesarios, siendo en la actualidad fuertemente resistidos por el Gobierno Central (sobre todo los Colegios fuertes que cuentan con Ley del Ejercicio Profesional como es el caso de médicos, abogados e ingenieros, entre otros, que agrupan, representan, articulan y defienden los intereses de su gremio).

Las asociaciones profesionales, por su parte, convocan psicólogos por áreas de interés y buscan organizar actividades de formación e intercambio científico. Actualmente, las más activas son: la Asociación Psicoanalítica de Estudios Lacanianos, la Sociedad Boliviana de Neuropsicología, la Asociación Científica de Psicología Forense, y la Asociación de Psicología Social de Bolivia (APISOBOL), (APISOBOL, s.f; Bejarano, 2007; Ocampo, 2009).

\section{Retos de la Psicología en Bolivia}

En este escenario, los retos para la Psicología a nivel profesional, de formación, y de desarrollo científico, son muchos.

Al ser una profesión joven, los profesionales todavía están abriéndose nuevos espacios en distintos sectores al tiempo que definen y defienden su campo de acción, porque la profe-

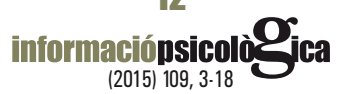


sión en La Paz tiene 40 años pero en la mayor parte del país, poco más de 20. Por ejemplo, según datos del CEUB, en 1997, había solo 182 psicólogos titulados en las universidades públicas de todo el país y 320 en la Universidad Católica Boliviana de La Paz (Universidad que abrió la primera carrera de Psicología del país en 1971).

Es así que los psicólogos que se encuentran en el sistema de asistencia social de los gobiernos departamentales y municipales, tropiezan con los espacios institucionalizados por los licenciados en Trabajo Social, que existen en Bolivia desde los años 40 y que presentaron dificultades para incorporar los informes psicológicos en los procesos que seguían y se resisten todavía a la inclusión de espacios de terapia e intervención. En el ámbito escolar, las funciones de los psicólogos y psicopedagogos se entremezclan, y fuera, algunos psicopedagogos consideran que pueden intervenir a nivel clínico. En el ámbito organizacional se encuentra a administradores de empresa aplicando pruebas en los procesos de selección y evaluación sin que haya normativa alguna que regule los instrumentos que se utilizan o quién tiene la formación para aplicarlos. En el campo de la salud, no hubo espacios institucionalizados para los psicólogos quienes trabajaron con ítems de Enfermería o Trabajo Social, hasta principios del 2015, cuando se promulgó una Resolución Ministerial del Ministerio de Salud (aún no publicada). Esto posibilitó que se les asigne ítem a algunos, desconociendo su antigüedad. Pero la resistencia a incluir psicólogos continúa, como se muestra en la publicación de ítems realizada en junio del 2015 para Santa Cruz, en la que no se requieren profesionales de la Psicología, a pesar de la demanda del sector y la presión realizada por el Colegio Departamental.

Sin contar con la falta de regulación y lo que esto implica en el ámbito clínico. Por tanto, los retos a este nivel, son enormes pero se van haciendo pequeños avances en la medida en la que la población de psicólogos crece y se va logrando una mayor articulación profesional a través de los Colegios de Psicólogos y asociaciones profesionales.

Sin embargo, en las regiones del país donde habían más proyectos de intervención social, antes de que el gobierno actual desautorice el funcionamiento de la cooperación internacional y ONG's, los requerimientos de profesionales del área social generalmente eran ocupados por psicólogos, incluso cuando se buscaba un perfil profesional de antropólogo, pedagogo o educador popular (J. López, comunicación personal, 14 de enero de 2014). Desgraciadamente este espacio laboral se ha visto mermado en el último tiempo.

Por otra parte, a nivel de formación y, por tanto, de formación docente, también hay mucho que hacer.

La estabilidad económica del último tiempo puede posibilitar algunos cambios. Para empezar, como estrategia política, el gobierno actual decidió destinar parte de los impuestos de los hidrocarburos a las universidades públicas, lo que ha multiplicado por más de diez su presupuesto anual permitiéndoles mejorar significativamente su infraestructura (“Gobierno emitió", 2012, 14 de agosto). Es de esperar (o al menos es deseable) que en los próximos años se dedique este dinero a formar el plantel docente y fomentar los centros de investigación. Así la percepción que la población tiene de su calidad mejorará, ya que por ahora se considera que la mejor formación se encuentra en las universidades privadas de mayor

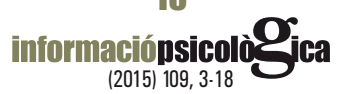




\section{dossier: Psicología lberoamericana - ||}

La Psicología en Bolivia: formación y situación laboral

costo (Universidad Católica Boliviana, Universidad Privada de Santa Cruz de la Sierra y Universidad Privada Boliviana, ésta última no ofrece la carrera de Psicología), (Contreras, 1998; Fundapro, 2011).

Los cambios que deben darse van más allá de la infraestructura, muchos de los cuales están directamente relacionados con la formación del plantel docente. Se observa que los docentes universitarios todavía sobrevaloran los libros y como hacen poco uso de las TICs tienden a basarse en textos antiguos, no solo por no estar muy interesados en buscar bibliografía que les permita contrastar teorías o cuestionar su conocimiento, sino porque ésta es muy difícil de encontrar en las librerías del país y porque durante su proceso formativo nadie les enseñó a buscar en otras fuentes de información. El acceso a material actualizado a través de Internet debería solucionar este problema, porque el acceso a la red no es privativo, pero se debe fomentar la alfabetización digital y la competencia informacional (enseñarles a localizar, evaluar y utilizar la información). Desgraciadamente, aunque bases de datos como EBSCO y HINARI están disponibles para las universidades, los docentes no las saben utilizar ni conocen la importancia de la información que contienen. Según un estudio realizado por Gainsborg (2013), los docentes utilizan la tecnología para realizar búsquedas de información para la docencia, pero estas suelen ser búsquedas superficiales de motores no especializados, siendo pocos los que hacen uso de reservorios de investigaciones indexadas, revistas académicas, etc. Es alentador, sin embargo, encontrar que los docentes dicen tener una actitud positiva hacia el uso de TICs en el aula lo que haría posible su alfabetización digital y el lograr que las utilicen como recurso pedagógico y de investigación (Gainsborg, 2013; Sainz, 2009). Lo cual queda como tarea para las instituciones de Educación Superior.

Las limitaciones que existen en la formación se hacen evidentes en la investigación. Así como tradicionalmente la producción científica latinoamericana está alejada de la producción de los países con mayor desarrollo científico y tecnológico, la producción científica en Psicología en Bolivia está alejada de la producción latinoamericana. Bolivia es uno de los dos países con menor producción científica, en Psicología, por millón de habitantes, en base a los datos obtenidos por Vera-Villarroel, López-López, Lillo y Silva (2011). Uno puede querer explicar la situación planteando las limitaciones impuestas por la eterna inestabilidad social, política y ecónomica, que deriva en infraestructura precaria, carencia de material bibliográfico, y en general, por la gran cantidad de restricciones que tienen las universidades, pero también conviven con ello los países vecinos y sus niveles de producción son otros. Por ejemplo, recurriendo a la tabla de IDH como indicador, se observa que Colombia está unos números por encima de Bolivia, es un país con 4,5 veces la población boliviana, sin embargo, tiene 37,5 veces más publicaciones en Psicología. Y eso no se debe solo a factores económicos, sino a todo un contexto cultural en el que la investigación y su fomento ha estado presente. El poco interés que se ha prestado, en Bolivia, a la investigación - en general - se observa haciendo un breve repaso por la historia de las entidades dirigidas a promoverla.

Por ejemplo, en 1860 el presidente de la República Dr. José María Linares fundó la primera Academia de Ciencias, y 100 años después, en 1960 el gobierno del Dr. Victor Paz Estensoro fundó la actual Academia de Ciencias de Bolivia, que se mantiene con un pequeño monto

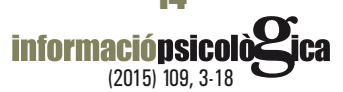




\section{dossier: Psicología lheroamericana - ||}

Marion K. Schulmeyer

del gobierno que cubre un personal mínimo y con apoyo de organizaciones internacionales. Los académicos que la componen no reciben ningún tipo de retribución monetaria. A partir del 2009, la Universidad Privada de Santa Cruz de la Sierra sostiene la Academia Nacional Ciencias de Bolivia - Departamental Santa Cruz y financia sus investigaciones (Academia Nacional de Ciencias de Bolivia, 2012).

En 1977 el Decreto Supremo N ${ }^{\circ}$ 15111, dió lugar al primer marco político, legal y normativo para la ciencia, tecnología y la innovación y creó el Consejo Nacional de Desarrollo Científico y Tecnológico y la Dirección de Ciencia y Tecnología, dependiente del Ministerio de Planeamiento y Coordinación, pero por cambios políticos, no avanzó y se quedó en papel. En 1991 se firmó el Decreto Supremo No 22908 que definía el Sistema Nacional de Ciencia y Tecnología, creó nuevamente el Consejo Nacional de Desarrollo Científico y Tecnológico (CONACYT), estableció políticas financieras y presupuestarias, y creó el Fondo Nacional de Ciencia y Tecnología. Esta vez, dependiendo del Vicepresidente de la República. Finalmente, en el 2001 se aprobó la Ley de Fomento de la Ciencia, Tecnología e Innovación. Y desde ese momento el Viceministerio de Ciencia y Tecnología realiza actividades con distintas entidades para socializar las líneas de investigación (Schulmeyer, 2013). Hasta el momento, pasó más de siglo y medio, y la ciencia sigue, en gran parte, definiendo sus intenciones. Hasta el momento, no ha habido fondos concursables para la investigación en el país, y cuando los haya, solo podran acceder a ellos las universidades y entes públicos.

Aún así, algunas universidades, en base a las iniciativas docentes, han ido creando centros de investigación, como son: el Instituto de Investigaciones en Ciencias del Comportamiento (IICC), de la Universidad Católica Boliviana, del cual se encuentran trabajos publicados desde el 2007 (UCB, 2013), el Instituto de Investigación e Interacción y Postgrado de Psicología (IIIPP) de la Universidad Mayor de San Andrés, creado el 2007 (Camacho, s.f.), y de creación más reciente, el Centro de Investigación y Asesoramiento Psicológico, (CIAP) de la Universidad Privada de Santa Cruz de la Sierra que funciona como tal desde el 2013 y desde el 2015 alberga el primer Laboratorio de Neurocognición.

\section{Conclusión}

La situación de la Psicología, como una carrera joven en Bolivia, hace que en la actualidad se continúen abriendo nuevos espacios para los profesionales, en los que se van definiendo los límites y áreas de actuación con otras profesiones en el área clínica, educativa, social y organizacional, pudiendo considerarse como un área laboral que va creciendo.

Las necesidades de formación del plantel docente en las universidades están haciendo que estas inviertan en ello. La exigencia del CEUB y del Ministerio de Educación ha hecho que todos los docentes al menos tengan un nivel de Diplomado en Educación Superior. Y que algunas universidades apoyen o subvencionen los programas de maestría y doctorado de sus docentes como es el caso de la Universidad Privada de Santa Cruz de la Sierra, que además les financia cursos de inglés a los que lo necesitan.

Es de esperar que la existencia actual de recursos económicos en las universidades públicas y sus procesos de acreditación redunden en niveles de formación más altos de los docentes, su actualización y apertura a la reflexión y a la producción. Los docentes de la universidad

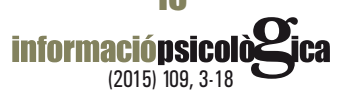


pública, en la actualidad son los mejor pagados, pero aun así, la mayor parte de los docentes son profesores a tiempo parcial que circulan de una a otra universidad, por lo que se espera que el esfuerzo que haga cualquier centro de estudios por formar a su plantel docente repercuta en las demás, y en la calidad de la educación de los futuros psicólogos.

Es así, que en un contexto difícil, la Psicología enfrenta retos, probablemente mucho más básicos que los que pueda tener en países donde está más desarrollada, pero se encuentra en un momento oportuno para mejorar. Se acaban de iniciar procesos de mejoramiento en las universidades, la sociedad está demandando la presencia de psicólogos en más espacios laborales, existe interés en el actual Viceministerio de Ciencia y Tecnología por capacitar a los docentes en investigación y en las universidades por elevar sus indicadores, en general (retención, graduación, formación docente, etc.), por lo que se puede esperar que en los próximos años este escenario sea otro. De hecho, desde que se escribió este artículo a cuando se revisó, la realidad cambió y exigió incluir algunos de los cambios que se dieron la primera mitad del 2015.

\section{Referencias}

Academia Nacional de Ciencias de Bolivia. (2012). Antecedentes. Origen y organización de la Academia. Recuperado el 23 de agosto de 2013, de http://www.aciencias.org.bo/antecedentes

Aguilar, G. (1983). Historia de la Psicología en Bolivia. Revista Latinoamericana de Psicología, 15(3), 311-325.

Bejarano, G. (2007). Historia de la Psicología Jurídica y Forense en Bolivia. La Paz: (no publicado).

Brañez, I. M. (2012). Colegio Departamental de Psicólogos de La Paz. En U. L. Salle, Desarrollo e historia de la psicología en la ciudad de La Paz (pág. 7). La Paz: Universidad La Salle.

Calderón, R. (1999). La psicología en Bolivia. En M. Alonso, Psicología en las Américas (págs. 47-65). Caracas: SIP.

Camacho, C. (s.f.). Historia del IIICC. Recuperado el 15 de enero de 2013, del Instituto de Investigación, Interacción y Postgrado de Psicología.: http://iiipp.psicologia.umsa.bo/iiipp/app;jsessionid=3AE0D8C4CF1854D065E 54B70EC751A4D?service $=$ external/AreaView\&sp=154009

Centro Interuniversitario de Desarrollo. (2006). Repitencia y deserción universitaria en América Latina. Santiago: CINDA- IESALC/UNESCO.

CEUB. (1997, octubre). Conclusiones de la reunión sectorial de la carrera de: Psicología. Recuperado el 23 de julio de 2014, de http://ceub.edu.bo/gaceta/img/sectoriales/41_II_IX_R_Psicologia.pdf

CEUB. (1998, septiembre). Conclusiones de la reunión sectorial de la Carrera de Trabajo Social. Recuperado el 23 de julio de 2014, de http://ceub.edu.bo/gaceta/img/sectoriales/39_II_IX_R_Trabajo_Social.pdf

CEUB. (2014, 23 de junio). Resoluciones. III Conferencia Nacional ordinaria de Universidades. 11-12 de mayo de 2011- Universidad Amazónica de Pando. Recuperado el 21 de julio de 2014, de http://www.ceub.edu. bo/index.php?option=com_content\&view=article\&id=54\&ltemid=61

Contreras, M. (1998). Reflexiones sobre la educación universitaria privada en Bolivia. Ciencia y Cultura, 3, 86-96.

Datax. (2012). Datax. Toda la información estadística de Bolivia. Recuperado el 30 de mayo de 2014, de http:// www.datax.com.bo/

Decreto Supremo No.15111. Sistema Nacional para el Desarrollo Científico y Tecnológico (SINDECYT). Gaceta Oficial de Bolivia. 
Decreto Supremo No.22908. Sistema Nacional de Ciencia y Tecnología. Gaceta Oficial del Estado Plurinacional de Bolivia.

Fundación para la Producción. (2007). Estudio del Mercado Laboral en Bolivia. La Paz: Autor.

Fundación para la Producción. (2011). Estudio del Mercado Laboral en Bolivia. Santa Cruz: Autor.

Gainsborg, C. (2013). Modelo de alfabetización digital para profesores universitarios a partir de un estudio empírico: Caso Universidad Privada de Santa Cruz de la Sierra. Trabajo no publicado. Facultad de Educación. Departamento de Teoría e Historia de la Educación. Universidad de Salamanca.

Gobierno emitió tres Decretos para que universidades usen 15\% de IDH. (2012, 14 de agosto). Jornada, La Paz.

Herrera, P. (1994). Sistemas de calidad en las Universidades Privadas de Bolivia. Un proyecto de ley justificado. Santa Cruz: Documento no publicado de la Fundación Libertad, Democracia y Desarrollo.

INE. (2011). INE: Población matriculada en la universidad. Recuperado el 21 de julio de 2014, de http://www. ine.gob.bo/indice/EstadisticaSocial.aspx? codigo $=30203$

Ley de Reforma Educativa. No. 1565 del 7 de julio de 1994. Obtenida en http://www.filosofia.org/mfa/fabo994a.htm Ministerio de Educación. (2011). Guía de Universidades del Estado Plurinacional de Bolivia 2011. La Paz: Autor.

Ministerio de Planificación y Desarrollo. (2009). Sistema Boliviano de Innovación. Viceministerio de Ciencia y Tecnología. Obtenido el 23 de julio de 2014 en: http://www.cienciaytecnologia.gob.bo/vcyt2012/ uploads/docsbi-01-06-2009.pdf.

Nueva Constitución Política del Estado. (2009). Gaceta Oficial de Bolivia. www.gacetaoficialdebolivia.gob.bo.

Ocampo, N. (2009). La neuropsicología en Bolivia. Revista Neuropsicología, Neuropsiquiatría y Neurociencias, 9(2), 29-33.

PNUD. (2013). Informe sobre Desarrollo Humano 2013. El ascenso del Sur: Progreso humano en un mundo diverso. Nueva York: Autor.

Puche, R. (2003). Elementos relevantes para pensar un "estado del arte de la psicología académica" en Colombia. ASCOFAPSI.

Rivera, E. A. (2006). Repitencia y deserción en la educación universitaria en Bolivia. En CINDA, Repitencia y deserción universitaria en América Latina. Santiago: CINDA - I.

Rodríguez, G., y Weise, C. (2006). Educación Superior Universitaria en Bolivia. Estudio nacional. Cochabamba: IESALC-UNESCO.

Sainz, P. (2009). Actitudes del Profesorado con Respecto al Uso de Tecnologías de la Información Comunicación en la Enseñanza Superior. Tesis de Maestría del Máster TIC en Educación: Análisis y diseño de procesos, recursos y prácticas formativas. Universidad de Salamanca.

Schulmeyer, M. K. (2013). 20 años de psicología en las Universidades Privadas de Bolivia. Ajayu, 11 (2), 118-137.

Schulmeyer, M. K. (2013, octubre). La investigación en Bolivia. Políticas y líneas de investigación. Conferencia presentada en el I Congreso Internacional de Investigación de la Universidad Nacional Federico Villarreal. Lima, Perú

Schulmeyer, M. K. (2014a). Carreras de Psicología y formación en Bolivia. En T. Caycho, Historia de la Psicología en Latinoamérica. Lima: Fondo Editorial Universidad Inca Garcilaso de la Vega. Manuscrito presentado para publicación.

Schulmeyer, M. K. (2014b). La psicometría en Bolivia. (Trabajo de investigación no publicado). Academia Nacional de Ciencias de Bolivia.

Unidad de Análisis de Políticas Sociales y Económicas. (2004). Informe Técnico Cálculo de las Líneas de Pobreza. UDAPE. Recuperado el 18 de junio de 2014 en http://www.eclac.cl/deype/mecovi/docs/TALLER13/6. pdf.

Universidad Autónoma Gabriel René Moreno. (1997, abril). / Seminario nacional sobre experiencias de evaluación en Bolivia. Santa Cruz: Autor. 
Universidad Católica Boliviana. (2013). Universidad Católica Boliviana "San Pablo". Obtenido el 10 de enero de 2013 de Unidad Académica Regional La Paz: http://lpz.ucb.edu.bo/Forms/Index.aspx

Universidad Privada de Santa Cruz de la Sierra. (2000). Memoria Anual 1999. Santa Cruz: Autor.

Universidad Privada de Santa Cruz de la Sierra. (2014). Certificaciones y Acreditaciones. Recuperado el 17 de julio de 2014, de Universidad Privada de Santa Cruz de la Sierra: https://www.upsa.edu.bo/index.php/es/ quienes-somos/certificaciones-y-acreditaciones

Vera-Villarroel, P., López-López, W., Lillo, S., y Silva, L. (2011). La producción científica en psicología latinoamericana: Un análisis de la investigación por países. Revista Latinoamericana de Psicología, 43 (1), 95-104.

Via, F. (2000). Historia y formación del psicólogo en Bolivia. Revista Ciencia y Cultura, 8, 51-62.

Weise, C. (2005). La construcción de políticas públicas universitarias en el período neoliberal. Estado y Universidad, contradicciones en una década de desconcierto: El caso de Bolivia. Tesis para optar al grado de Magíster en Ciencias Sociales con mención en Educación: FLACSO. 